\title{
Situación de salud de gestantes migrantes venezolanas en el Caribe colombiano: primer reporte para una respuesta rápida en Salud Pública
}

\section{Health situation of Venezuelan pregnant migrants in the Colombian Caribbean: first report for a rapid response in Public Health}

\author{
Julián-Alfredo Fernández-Niño; ${ }^{1}$ Maylen-Liseth Rojas-Botero²; Ietza Bojorquez-Chapela ${ }^{3}$; Vanesa Giraldo-Gartner; \\ Rita-Aleksandra Sobczyk ${ }^{1}$; Jorge Acosta-Reyes ${ }^{1}$; Víctor Flórez-García ${ }^{1}$; David-Alejandro Rodríguez ${ }^{5}$
}

Forma de citar: Fernández-Niño JA, Rojas-Botero ML, Bojorquez-Chapela I, Giraldo-Gartner V, Sobczyk RA, Acosta-Reyes J, et al. Situación de salud de gestantes migrantes venezolanas en el Caribe colombiano: primer reporte para una respuesta rápida en Salud Pública. Rev Univ Ind Santander Salud. 2019; 51(3): 208-219. doi: http://dx.doi.org/10.18273/revsal.v51n3-2019004 (c) (1)

\section{Resumen}

Introducción: En el fenómeno migratorio desde Venezuela hacia Colombia, las mujeres representan una proporción significativa entre los migrantes irregulares; de ellas, las gestantes han sido priorizadas por sus condiciones particulares de vulnerabilidad. Sin embargo, se desconocen sus condiciones de salud. Objetivos: Caracterizar multidimensionalmente la situación de salud de las gestantes venezolanas migrantes irregulares, residentes en las principales ciudades receptoras del Caribe colombiano: Barranquilla y Riohacha. Metodología: Estudio descriptivo de corte transversal en 520 gestantes venezolanas migrantes irregulares, captadas por muestreo sistemático en hospitales públicos, y comunitariamente a través de búsqueda activa en bola de nieve entre agosto de 2018 y mayo de 2019. Con un cuestionario estandarizado se evaluaron los principales problemas de salud, estado nutricional, seguridad alimentaria, presencia de síntomas depresivos, accesibilidad y satisfacción con los servicios de salud. Resultados: La mitad percibía su salud durante el embarazo como buena o muy buena (53,4\%). El 51,3\% presentó anemia ferropénica y la mayoría algún grado de inseguridad alimentaria (87,5\%). La prevalencia de síntomas depresivos clínicamente significativos fue del 32,1\%. Una alta proporción reportó algún tipo de violencia por parte de su pareja (90,2\%). El 25,9\% requirió atención sanitaria por urgencias independiente de la atención prenatal. El $62,7 \%$ calificó la atención por urgencias como muy buena o buena, mientras que el 73,1\% piensa que le brindaron la atención necesaria para su problema de salud. Conclusiones: Los principales problemas de salud en gestantes que participaron en el estudio corresponden a la inseguridad alimentaria, anemia, síntomas depresivos, violencia de pareja y ausencia de controles prenatales. Su situación de salud debe considerarse en el marco del proceso migratorio y desde los determinantes sociales de la salida, tránsito, llegada y retorno. Las medidas de atención primaria para esta población deben seguir siendo fortalecidas por el sistema de salud a nivel territorial y basadas en evidencia.

Palabras clave: Emigración e inmigración; Salud materna; Determinantes sociales de la salud; Venezuela; Colombia; Salud reproductiva; Inseguridad alimentaria; Embarazo; Cuidado prenatal; Región Caribe.

1. Universidad del Norte. Barranquilla, Colombia.

2. Universidad de Antioquia. Medellín, Colombia.

3. El Colegio de La Frontera Norte. Tijuana, México

4. University of Massachussets. Boston, Estados Unidos.

5. Programa Migración y Salud, Organización Internacional para las Migraciones. Bogotá, Colombia.

Correspondencia: Maylen Liseth Rojas Botero. Dirección: Calle 62. 52-59 Medellín. Teléfono: +54 421 96820. Correo electrónico: maylen. rojas@udea.edu.co 


\begin{abstract}
Introduction: In the current migration from Venezuela to Colombia, women represent a significant proportion of the irregular migrant population, and those who are pregnant have been a priority given their particularly vulnerable condition. Nevertheless, their health conditions are not known. Objectives: To obtain a multi-dimensional characterization of the health situation of irregular Venezuelan migrants who are pregnant and residing in the main receiving the Caribbean region of Colombia: Barranquilla and Riohacha. Methods: We carried out a descriptive, cross-sectional study with 520 pregnant women surveyed from August 2018 to May 2019 with a systematic sampling in public hospitals and an active community search using snowball sampling. We used a standardized questionnaire to evaluate the main health problems, nutritional status, food security, presence of depressive symptoms and accessibility/ satisfaction with health services. Results: Half of the participants perceived their health during pregnancy as good or very good (53.4\%), 51.3\% presented iron deficiency anemia, and the majority $(87.5 \%)$ experienced some lack of food security. The prevalence of clinically significant depressive symptoms was $32.1 \%$. A high proportion reported some type of intimate partner violence (90.2\%). 25.9\% required urgent medical care, irrespective of the prenatal care they received. With regard to quality of care in the emergency service, $62.7 \%$ rated it as very good or good, and $73.1 \%$ reported that they received the care that was needed for their health problem. Conclusions: Food insecurity, anemia, depressive symptoms, violence committed by the partner and lack of prenatal care were the main health problems for pregnant women who participated in the study. Their health situation should be viewed in the context of the migration process, based on the social determinants of emigration, transit, arrival, and return. The health care system should continue to strengthen primary care measures for this population at the local level and based on the evidence.
\end{abstract}

Keywords: Emigration and Immigration; Maternal health; Social determinants of health; Venezuela; Colombia; Reproductive health; Food supply; Pregnancy; Prenatal care; Caribbean region.

\section{Introducción}

En Colombia hay actualmente más de un millón de migrantes venezolanos, de los cuales, cerca de la mitad son irregulares o están en vía de regularización, y de ellos, aproximadamente la mitad son mujeres en edad reproductiva ${ }^{1}$. Este fenómeno migratorio contrasta con el perfil migratorio tradicional colombiano, dado que el país históricamente había sido un país emisor de personas y sólo hasta los últimos años se ha convertido también en uno de los principales países receptores de la migración sursur de la región ${ }^{2}$, en especial desde la agudización de la crisis política y económica de Venezuela; así, actualmente Colombia es el principal receptor de migrantes venezolanos irregulares.

Durante los últimos años en el país se ha observado un marcado incremento en la prestación de servicios sanitarios para migrantes, lo cual es explicado significativamente por la atención prenatal y la atención del parto y puerperio de gestantes venezolanas. De esta manera, en 2018 más de tres mil partos de migrantes venezolanas fueron atendidos en la red pública hospitalaria del país, un incremento de cuatro veces con respecto al año anterior ${ }^{3}$, lo cual constituye un reto financiero y de gestión en Salud Pública para los sistemas de salud a nivel nacional y regional ${ }^{4,5}$.
Las relaciones entre migración, gestación y salud son complejas. La gestación puede ser circunstancial al proceso migratorio como intersección del ciclo vital de la mujer migrante, pero también en algunos casos puede por sí misma motivar la migración en búsqueda de mejor atención en los servicios de salud ${ }^{6,7}$, en especial en un contexto de escasez de alimentos y medicamentos, como el que sucede en Venezuela, siendo este aparentemente el proceso en la frontera. Las migrantes gestantes, en especial las irregulares, son expuestas a riesgos psicosociales y ambientales durante su proceso de tránsito y llegada, cargan con las exposiciones relevantes del lugar de origen, y además enfrentan diversos y nuevos riesgos para su salud en el lugar de destino ${ }^{8}$. Adicionalmente, pueden tener dificultades para enfrentar sus necesidades de salud dado que no siempre conocen sus derechos y las rutas de acceso al sistema de salud colombiano.

En el marco del reciente fenómeno migratorio proveniente desde Venezuela hacia Colombia, convencidos de la importancia de la evidencia para la toma de decisiones y la construcción de políticas públicas, la Universidad del Norte en convenio con la Organización Internacional para las Migraciones (OIM) gestionó un estudio para evaluar el estado de salud de las gestantes migrantes irregulares de forma multidimensional y su experiencia con los servicios sanitarios en el país, de acuerdo con aspectos 
demográficos, sociales y económicos, con el propósito de aportar información para el desarrollo de estrategias de atención primaria para vigilar, promover y proteger la salud de las gestantes migrantes en Colombia. En este artículo se presenta el primer informe derivado de los resultados de la primera ola de recolección de información, correspondiente a las migrantes irregulares asentadas en Barranquilla y Riohacha entre 2018 y 2019, principales ciudades receptoras del caribe colombiano.

\section{Metodología}

Diseño de estudio: Se llevó a cabo un estudio de corte transversal con fuente primaria, de base hospitalaria y comunitaria, en Barranquilla y Riohacha, entre julio de 2018 y mayo de 2019. Población blanco de estudio y criterios de selección: gestantes venezolanas en condición irregular, asentadas en el Caribe colombiano durante al menos dos meses que aceptaron participar en el estudio. Se consideraron las pacientes atendidas en los hospitales públicos y las mujeres de base comunitaria que pudieran o no haber tenido contacto con los servicios de salud al momento de la evaluación. De las anteriores, se excluyeron quienes tenían nacionalidad colombiana.

Muestreo: Marco muestral: Se llevó a cabo diferencialmente para las pacientes de base hospitalaria y para las gestantes de base comunitaria. Para las de base hospitalaria el reclutamiento se hizo en el punto de atención de la red pública hospitalaria Camino Simón Bolívar y en el Hospital Nazareth de Barranquilla. En Riohacha la búsqueda se hizo en el Hospital Nuestra Señora de los Remedios (E.S.E.). Estos hospitales corresponden a las instituciones designadas por las autoridades sanitarias para atender los controles prenatales de las gestantes migrantes irregulares. Otras unidades de la red pública de Barranquilla atienden exclusivamente urgencias, por lo que no se consideraron.

En el caso de Riohacha, debido a cambios en la atención de las gestantes, el reclutamiento se hizo en urgencias o en un puesto de atención dispuesto por una Organización No Gubernamental dentro del Hospital, dado que hubo periodos sin prestación de servicio para control prenatal.

Por su parte y también en ambas ciudades, para la búsqueda comunitaria se realizaron varias estrategias de rastreo activo, a saber: referencia de gestantes identificadas en el hospital, visita a cinco asentamientos de Barranquilla para búsqueda activa con el acompañamiento de líderes comunitarios, búsqueda propositiva a través de líderes y asociaciones de migrantes, convocatoria por redes sociales, rastreo en jornadas de vacunación o atención primaria lideradas por autoridades u organismos humanitarios. Para esta búsqueda se dispusieron de todos los recursos posibles en ambas ciudades. Se tuvo contacto con el $89,5 \%$ de las mujeres identificadas en la estrategia y todas aceptaron participar.

Procedimiento de muestreo y tamaño de muestra: Para la muestra de base hospitalaria, en cada institución se realizó un muestreo sistemático con base en numeración del orden de llegada, en horas de consulta (mañanas de lunes a viernes de 7 am a $12 \mathrm{~m}$ ); cada día se asignó un número a cada paciente y se seleccionó aleatoriamente un número de arranque (entre 1 a 5), usando esa distancia entre pacientes para invitarlas a participar el estudio. El 100\% de las gestantes de base hospitalaria aceptaron participar. El tamaño de muestra se estimó considerando la semi-amplitud de los intervalos de confianza mediante la siguiente fórmula:

$$
d=1,96 \sqrt{\frac{p(1-p)}{n}} * \text { Deff }
$$

Donde $\mathrm{p}$ es la prevalencia de interés y Deff el efecto de diseño. Este último (Deff) se interpreta como el número de unidades colectadas mediante esta encuesta que son equivalentes para propósitos de estimación a una unidad colectada mediante muestreo aleatorio simple. El valor de Deff estimado en esta encuesta fue de 1,83. Los parámetros que se estimarían en este estudio se supusieron en el intervalo de entre $10 \%$ al $70 \%$.

Dados los parámetros, las ganancias en precisión en la semi-amplitud de los intervalos son marginales para tamaños de muestra mayores a 500 (Figura 1); considerando una no respuesta del $5 \%$, el tamaño de muestra total estimado fue de 525 .

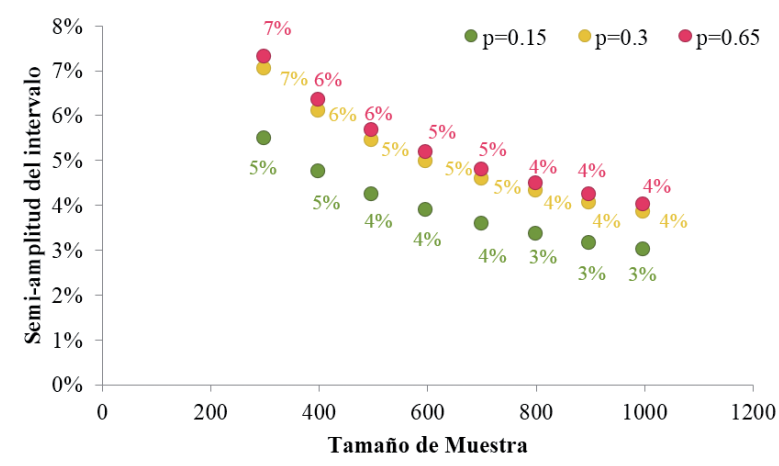

Figura 1. Semi-amplitud de los intervalos de confianza. 
Instrumento de recolección de Información: Se construyó un instrumento con 134 preguntas, a partir de cuestionarios de referencia como la Encuesta Nacional de Demografía y Salud (ENDS), la encuesta sobre Migración en la frontera Sur de México (Cuestionario 2016) y el instrumento del Instituto Sonorense de la Mujer (México 2010).

Se utilizó la Escala Latinoamericana y Caribeña de Seguridad Alimentaria (ELCSA) validada para Colombia9. Los puntos de corte para seguridad alimentaria provienen del manual de uso y aplicaciones ${ }^{10}$. Para indagar sobre los síntomas depresivos, se usó la escala del Centro de Estudios Epidemiológicos (CESD-D) con 21 ítems; cuando la gestante obtuvo 12 puntos o más se clasificó con síntomas depresivos clínicamente significativos ${ }^{11}$.

Adicionalmente, a una submuestra de 273 gestantes asentadas en Barranquilla, se les cuantificó la hemoglobina en sangre capilar. Para esto, se recolectó una muestra de sangre del dedo anular y se cuantificó la concentración de hemoglobina utilizando un hemoglobinómetro portátil. La lectura se realizó en dos longitudes de onda y $880 \mathrm{~nm}$ para compensar turbidez. Considerando un umbral fijo de anemia que genera mayor incidencia de problemas al nacimiento de $11 \mathrm{~g} / \mathrm{dl}^{12}$.

Pilotaje del instrumento: El cuestionario fue revisado y avalado por dos expertos internacionales en migración y salud, ajenos al equipo de trabajo. Antes de la aplicación, se realizó una prueba piloto en 20 gestantes en ambas ciudades para identificar eficacia y aceptabilidad del instrumento, así como necesidades ajustes de lenguaje o de revisión de opciones de respuesta. El instrumento fue ajustado después del piloto.

Control de calidad del dato: El cuestionario y la toma de muestra se realizó por profesionales de salud (enfermera y microbióloga) con experiencia en aplicación de encuestas, quienes fueron entrenadas en el instrumento, siguiendo un protocolo estricto estandarizado. Uno de los investigadores hizo supervisión semanal de la calidad del dato.

Análisis estadístico: Se realizó un análisis descriptivo con medidas de tendencia central y dispersión para las variables cuantitativas. Para el caso de las variables cualitativas se realizaron proporciones. Los resultados se presentan estratificadamente por ciudad y tipo de muestra. Se usó STATA 12 (Stata Corporation, College Station, TX, USA).

\section{Resultados}

\section{Características demográficas, sociales y económicas}

Fueron encuestadas 520 mujeres entre agosto de 2018 y mayo de 2019, la mayoría de ellas en Barranquilla $(60,8 \%)$ y en el entorno hospitalario $(81,3 \%)$. Se trata de mujeres jóvenes, predominantemente casadas o en unión libre, con compañero venezolano. En un $24,4 \%$ mujeres cabeza de familia, y con algún grado educativo (Tabla 1).

Ocho de cada diez pagaban arriendo por su vivienda en Colombia $(76,6 \%)$, la cual correspondía, principalmente, a una casa $(37,1 \%)$ o apartamento $(21,5 \%)$. En promedio residían en 2,1 habitaciones con 4,8 personas, de las cuales 1,8 percibían ingresos. En más de la mitad de los hogares el ingreso mensual no superaba el salario mínimo colombiano (que para 2019 estaba aproximadamente en US\$245) y durante el mes anterior a la encuesta, dicho dinero fue invertido casi totalmente en la compra de alimentos $(59,3 \%$ ) y el pago del arriendo (29,3\%); la principal fuente de ingresos de la gestante correspondía a los ingresos del cónyuge o pareja $(67,5 \%)$, seguido por el trabajo propio $(18,3 \%)$ y la colaboración de la familia $(11,3 \%)$. Casi la mitad de las encuestadas enviaba remesas a Venezuela (49,0\%).

En la mayoría de las viviendas las gestantes contaban con servicios públicos de energía eléctrica, acueducto y alcantarillado; más de la mitad tenía gas natural, mientras que el $12,3 \%$ no contaba con servicio de recolección de basuras, con menor disponibilidad entre las gestantes asentadas en Riohacha (Tabla 1).

\section{Características del proceso migratorio}

La mayoría de las gestantes encuestadas salieron de Venezuela en el año 2018 (59,2\%). Si bien casi todas habían elegido a Colombia como lugar de destino antes de migrar (Tabla 2), un 1,0\% planeaba llegar hasta otro país (Ecuador, Perú o USA). El 57,9\% se transportó en bus para llegar a Colombia, seguidas por quienes se movilizaron en automóvil (21,0\%).

El 9,6\% dijo haber contratado alguna persona para que le ayudara a cruzar la frontera, al 4,6\% amenazaron con llamar a las autoridades migratorias y el 5,2\% dijo recibir burlas, insultos o gritos durante su ingreso a Colombia. El 10,0\% refirió haber sido víctima de robo o atraco en el desplazamiento, el 1,3\% víctima de agresión física y $0,6 \%$ víctima de abuso sexual. 
Situación de salud de gestantes migrantes venezolanas en el Caribe colombiano: primer reporte para una respuesta rápida en Salud Pública

Tabla 1. Características sociodemográficas de gestantes venezolanas migrantes en condición irregular en Barranquilla y La Guajira, 2018-2019.

\begin{tabular}{|c|c|c|c|c|c|c|c|c|c|}
\hline & \multicolumn{3}{|c|}{ Barranquilla } & \multicolumn{3}{|c|}{ Riohacha } & \multicolumn{3}{|c|}{ Total } \\
\hline & Comu $^{\mathrm{a}}$ & Hosp $^{b}$ & Total & Comu & Hosp & Total & Comu & Hosp & Total \\
\hline \multicolumn{10}{|l|}{ Edad } \\
\hline $\begin{array}{l}\text { Media } \\
\left(\mathrm{DE}^{\mathrm{c}}\right)\end{array}$ & $\begin{array}{l}23,7 \\
(5,0)\end{array}$ & $\begin{array}{l}23,9 \\
(5,4)\end{array}$ & $\begin{array}{l}23,8 \\
(5,3)\end{array}$ & $\begin{array}{l}23,9 \\
(4,9)\end{array}$ & $\begin{array}{l}24,1 \\
(5,2)\end{array}$ & $\begin{array}{l}24,1 \\
(5,1)\end{array}$ & $\begin{array}{l}23,7 \\
(4,9)\end{array}$ & $\begin{array}{l}24,0 \\
(5,3)\end{array}$ & $\begin{array}{l}23,9 \\
(5,2)\end{array}$ \\
\hline 14 - 19 años, \% & 22,0 & 20,6 & 20,9 & 16,2 & 20,3 & 19,5 & 19,8 & 20,5 & 20,4 \\
\hline 20 - 34 años, $\%$ & 72,9 & 74,2 & 74,0 & 78,4 & 75,9 & 76,4 & 75,0 & 74,9 & 74,9 \\
\hline 35 - 41 años, \% & 5,1 & 5,2 & 5,1 & 5,4 & 3,8 & 4,1 & 5,2 & 4,6 & 4,7 \\
\hline \multicolumn{10}{|l|}{ Estado civil \% } \\
\hline Soltera & 16,9 & 11,3 & 12,3 & 55,3 & 11,4 & 19,6 & 32,0 & 11,3 & 15,2 \\
\hline Casada - Unión libre & 79,7 & 88,3 & 86,7 & 39,5 & 79,5 & 72,1 & 63,9 & 84,9 & 81,0 \\
\hline Separada - divorciada & 3,4 & 0,4 & 0,9 & 5,3 & 7,2 & 6,9 & 4,1 & 3,1 & 3,3 \\
\hline Viuda & 0,0 & 0,0 & 0,0 & 0,0 & 1,8 & 1,5 & 0,0 & 0,7 & 0,6 \\
\hline \multicolumn{10}{|l|}{ Nivel educativo \% } \\
\hline Ninguno & 0,0 & 0,4 & 0,3 & 0,0 & 1,8 & 1,5 & 0,0 & 0,9 & 0,8 \\
\hline Primaria & 16,9 & 25,3 & 23,8 & 42,1 & 33,7 & 35,3 & 26,8 & 28,7 & 28,3 \\
\hline Secundaria & 67,8 & 48,0 & 51,7 & 50,0 & 47,6 & 48,0 & 60,8 & 47,9 & 50,3 \\
\hline Técnico medio & 8,5 & 7,0 & 7,3 & 2,6 & 6,0 & 5,4 & 6,2 & 6,6 & 6,6 \\
\hline Técnico superior & 6,8 & 12,9 & 11,7 & 0,0 & 4,2 & 3,4 & 4,1 & 9,5 & 8,5 \\
\hline Pregrado & 0,0 & 6,3 & 5,1 & 5,3 & 6,6 & 6,4 & 2,1 & 6,4 & 5,6 \\
\hline \multicolumn{10}{|c|}{ Número de personas con las que reside } \\
\hline Media (DE) & $\begin{array}{c}6,5 \\
(4,4)\end{array}$ & $\begin{array}{c}4,7 \\
(2,7)\end{array}$ & $\begin{array}{c}5,1 \\
(3,2)\end{array}$ & $\begin{array}{c}3,4 \\
(2,0)\end{array}$ & $\begin{array}{c}4,6 \\
(2,8)\end{array}$ & $\begin{array}{c}4,4 \\
(2,7)\end{array}$ & $\begin{array}{c}5,3 \\
(4,0)\end{array}$ & $\begin{array}{c}4,7 \\
(2,8)\end{array}$ & $\begin{array}{c}4,8 \\
(3,0)\end{array}$ \\
\hline $1-6$ personas, $\%$ & 64,4 & 78,9 & 76,2 & 94,7 & 80,1 & 82,8 & 76,3 & 79,4 & 78,8 \\
\hline $7-12$ personas, $\%$ & 22,0 & 19,5 & 20,0 & 5,3 & 18,1 & 15,7 & 15,5 & 19,0 & 18,3 \\
\hline $13-21$ personas, $\%$ & 13,6 & 1,6 & 3,8 & 0,0 & 1,8 & 1,5 & 8,2 & 1,7 & 2,9 \\
\hline
\end{tabular}

Personas que reciben ingresos

Media (DE)

$2,0(1,5) \quad 1,8(1,1) \quad 1,9(1,2) \quad 1,6(0,9) \quad 1,6(1,3) \quad 1,6(1,3) \quad 1,9(1,3) \quad 1,8(1,2) \quad 1,8(1,2)$

Ingreso mensual total en el hogar $\%$

\begin{tabular}{|c|c|c|c|c|c|c|c|c|c|}
\hline Menos de 1 SMMLV & 67,8 & 63,8 & 64,6 & 81,6 & 66,9 & 69,6 & 73,2 & 65,0 & 66,5 \\
\hline Entre $1 \mathrm{y}<3,5$ SMMLV & 32,2 & 35,8 & 35,1 & 18,4 & 33,1 & 30,4 & 26,8 & 34,8 & 33,3 \\
\hline Entre $3,5 \mathrm{y}<6 \mathrm{SMMLV}$ & 0,0 & 0,4 & 0,3 & 0,0 & 0,0 & 0,0 & 0,0 & 0,2 & 0,2 \\
\hline \multicolumn{10}{|c|}{ cibe ayudas o subsidios económicos \% } \\
\hline Sí & 20,3 & 14 & 15,2 & 2,6 & 16,9 & 14,2 & 13,4 & 15,1 & 14,8 \\
\hline \multicolumn{10}{|c|}{ recibido asistencia o ayudas $\%$} \\
\hline Sí & 47,5 & 33,1 & 35,8 & 10,5 & 48,2 & 41,2 & 33,0 & 39,0 & 37,9 \\
\hline \multicolumn{10}{|l|}{ vía dinero a Venezuela \% } \\
\hline Sí & 50,8 & 50,8 & 50,8 & 34,2 & 49,1 & 46,3 & 44,3 & 50,1 & 49,0 \\
\hline \multicolumn{10}{|c|}{ rvicios públicos en el hogar $\%$} \\
\hline Energía eléctrica & 96,6 & 99,6 & 99,1 & 97,4 & 92,2 & 93,1 & 96,9 & 96,7 & 96,7 \\
\hline Gas natural & 54,2 & 79,8 & 75,0 & 65,8 & 50,0 & 52,9 & 58,8 & 68,1 & 66,3 \\
\hline Acueducto & 89,8 & 97,3 & 95,9 & 92,1 & 81,9 & 83,8 & 90,7 & 91,3 & 91,2 \\
\hline Alcantarillado & 67,8 & 95,3 & 90,2 & 92,1 & 74,1 & 77,5 & 77,3 & 87,0 & 85,2 \\
\hline Internet & 15,3 & 20,2 & 19,3 & 2,6 & 9,0 & 7,8 & 10,3 & 15,8 & 14,8 \\
\hline Recolección de basuras & 69,5 & 96,9 & 91,8 & 92,1 & 78,9 & 81,4 & 78,4 & 89,8 & 87,7 \\
\hline
\end{tabular}

a. Comu: Gestantes de base comunitaria

b. Hosp: Gestantes de base hospitalaria

c. DE: Desviación estándar 
Tabla 2. Características del proceso migratorio de gestantes venezolanas en condición irregular en Barranquilla y La Guajira, 2018-2019.

\begin{tabular}{|c|c|c|c|c|c|c|c|c|c|}
\hline & \multicolumn{3}{|c|}{ Barranquilla } & \multicolumn{3}{|c|}{ Riohacha } & \multicolumn{3}{|c|}{ Total } \\
\hline & Comu & Hosp & Total & Comu & Hosp & Total & Comu & Hosp & Total \\
\hline \multicolumn{10}{|l|}{ Motivos para migrar \% } \\
\hline Represión económica & 78,0 & 78,5 & 78,4 & 86,8 & 65,1 & 69,1 & 81,4 & 73,2 & 74,8 \\
\hline Mejor futuro & 8,5 & 16,4 & 14,9 & 10,5 & 18,1 & 16,7 & 9,3 & 17,1 & 15,6 \\
\hline Razones familiares & 5,1 & 1,2 & 1,9 & 2,6 & 9,0 & 7,8 & 4,1 & 4,3 & 4,2 \\
\hline Represión política & 3,4 & 0,8 & 1,3 & 0,0 & 5,4 & 4,4 & 2,1 & 2,6 & 2,5 \\
\hline Seguridad (no económica) & 3,4 & 0,4 & 1,0 & 0,0 & 0,6 & 0,5 & 2,1 & 0,5 & 0,8 \\
\hline Otro & 1,7 & 2,7 & 2,5 & 0,0 & 1,8 & 1,5 & 1,0 & 2,4 & 2,1 \\
\hline \multicolumn{10}{|c|}{ Tiempo de estancia en Colombia en meses } \\
\hline Media (DE) & $\begin{array}{r}11,3 \\
(6,6)\end{array}$ & $\begin{array}{l}12,7 \\
(7,5)\end{array}$ & $\begin{array}{l}12,4 \\
(7,4)\end{array}$ & $\begin{array}{c}9,9 \\
(5,3)\end{array}$ & $\begin{array}{l}11,0 \\
(8,1)\end{array}$ & $\begin{array}{l}10,8 \\
(7,6)\end{array}$ & $\begin{array}{l}10,8 \\
(6,1)\end{array}$ & $\begin{array}{l}12,0 \\
(7,8)\end{array}$ & $\begin{array}{l}11,8 \\
(7,5)\end{array}$ \\
\hline $2-6$ meses $\%$ & 30,5 & 19,5 & 21,5 & 23,7 & 27,7 & 27 & 27,8 & 22,7 & 23,7 \\
\hline $7-12$ meses $\%$ & 40,7 & 47,1 & 45,9 & 68,4 & 47,6 & 51,5 & 51,5 & 47,3 & 48,1 \\
\hline $13-24$ meses $\%$ & 28,8 & 30,0 & 29,7 & 2,6 & 16,9 & 14,2 & 18,6 & 24,8 & 23,7 \\
\hline Más de 24 meses \% & 0,0 & 3,5 & 2,8 & 5,3 & 7,8 & 7,4 & 2,1 & 5,2 & 4,6 \\
\hline \multicolumn{10}{|c|}{ Tiempo de estancia en Barranquilla - Riohacha en meses } \\
\hline Media (DE) & $\begin{array}{l}10,8 \\
(6,6)\end{array}$ & $\begin{array}{l}11,7 \\
(7,3)\end{array}$ & $\begin{array}{l}11,5 \\
(7,1)\end{array}$ & $\begin{array}{c}9,9 \\
(5,3)\end{array}$ & $\begin{array}{l}10,3 \\
(7,6)\end{array}$ & $\begin{array}{l}10,3 \\
(7,2)\end{array}$ & $\begin{array}{l}10,4 \\
(6,1)\end{array}$ & $\begin{array}{l}11,2 \\
(7,4)\end{array}$ & $\begin{array}{l}11,0 \\
(7,2)\end{array}$ \\
\hline $2-6$ meses $\%$ & 35,6 & 23,7 & 25,9 & 23,7 & 28,9 & 27,9 & 30,9 & 25,8 & 26,7 \\
\hline $7-12$ meses $\%$ & 37,3 & 47,5 & 45,6 & 68,4 & 49,4 & 52,9 & 49,5 & 48,2 & 48,5 \\
\hline $13-24$ meses $\%$ & 27,1 & 26,1 & 26,3 & 2,6 & 15,1 & 12,7 & 17,5 & 21,7 & 21 \\
\hline Más de 24 meses \% & 0,0 & 2,7 & 2,2 & 5,3 & 6,6 & 6,4 & 2,1 & 4,3 & 3,8 \\
\hline \multicolumn{10}{|c|}{ Había decidido venir a Colombia antes de salir de Venezuela \% } \\
\hline Sí & 96,6 & 94,6 & 94,6 & 86,8 & 84,3 & 84,8 & 92,8 & 90,5 & 91,0 \\
\hline \multicolumn{10}{|l|}{ Participó en el RAMV \% } \\
\hline Sí & 44,1 & 29,6 & 32,3 & 50,0 & 41,6 & 43,1 & 46,4 & 34,3 & 36,5 \\
\hline \multicolumn{10}{|l|}{ País donde planea el parto \% } \\
\hline Colombia & 98,3 & 97,2 & 97,4 & 97,4 & 96,4 & 96,6 & 97,9 & 96,9 & 97,1 \\
\hline Venezuela & 1,7 & 2,4 & 2,2 & 2,6 & 3,6 & 3,4 & 2,1 & 2,9 & 2,7 \\
\hline Otro & 0,0 & 0,4 & 0,3 & 0,0 & 0,0 & 0,0 & 0,0 & 0,2 & 0,2 \\
\hline \multicolumn{10}{|l|}{ Planea retornar a Venezuela \% } \\
\hline Sí & 52,5 & 60,7 & 59,2 & 21,1 & 37,3 & 34,3 & 40,2 & 51,5 & 49,4 \\
\hline
\end{tabular}

\section{Condiciones de salud y acceso a los servicios sanitarios}

Al momento de la encuesta el 50\% de las mujeres tenía seis meses de gestación o menos ( $\mathrm{Rq}=4$ meses) y en el $1,0 \%$ de los casos se trataba de un embarazo múltiple. El 42,0\% de las encuestadas eran primerizas, las demás tenían en promedio 2,0 hijos ( $\mathrm{DE}=1,2$ hijos).

Entre los antecedentes obstétricos referidos (entre quienes tenían hijos), se destacaron la cesárea anterior $(37,6 \%)$, las complicaciones durante el embarazo
$(36,0 \%)$, parto pretérmino $(16,2 \%)$, ruptura prematura de membranas $(10,8 \%)$, bajo peso al nacer $(7,1 \%)$ y malformaciones congénitas $(2,7 \%)$.

Durante este embarazo, cuatro de cada diez percibían su propia salud como regular $(40,2 \%)$ con peor percepción para las gestantes de Riohacha (Tabla 3); el 26,7\% había presentado al menos un episodio de infección urinaria, el 5,6\% tenía diagnóstico de preeclampsia, el $3,1 \%$ de enfermedades bucodentales, el $1,5 \%$ de diabetes gestacional y un $17,8 \%$ adicional que refirió otra enfermedad sin especificar. Se encontró que el 
23,4\% de las gestantes presentaba síntomas depresivos clínicamente significativos (con una diferencia de 21,4 pp entre las ciudades, con mayor proporción para las asentadas en Riohacha), mientras el 16,6\% consideraba que estar embarazada era un problema.

Entre tanto, al 23,0\% le habían informado que el embarazo actual era de alto riesgo debido a su edad, antecedente de abortos, diabetes gestacional, sífilis, hepatitis B, cardiopatía, epilepsia, trombosis venosa profunda, ruptura de arteria femoral, obesidad, embarazo ectópico anterior, captación tardía, preeclampsia, desprendimiento de placenta, placenta previa y cordón en el cuello. De otro lado, al 0,7\% tenía diagnóstico de anomalía cromosómica, en este caso, anomalías cardiacas.

Solo en el 3,7\% de los casos refirieron estar afiliadas al sistema de salud colombiano, todas en el régimen subsidiado, excepto una que dijo estar en el régimen contributivo. La institución donde recibían la atención sanitaria estaba a mínimo 2 minutos y máximo a 2 horas de distancia y la mayoría se trasladaba en bus $(37,1 \%)$ o en mototaxi $(26,6 \%)$.

Solo al 32,3\% le habían otorgado un carné materno para la atención en salud, mientras que dos de cada cinco o menos habían recibido las pruebas prenatales y vacunas necesarias, así: vacuna contra influenza, 44,9\%; vacuna contra tétanos, 46,8\%; ecografías, 37,3\%; exámenes de laboratorio, 38,4\%; hemoclasificación, 28,9\%; y prueba de tolerancia a la glucosa, 28,0\%. En este escenario, la prueba de VIH y sífilis solo fue reportada por el $33,2 \%$ y el $31,8 \%$, respectivamente, es decir, se desconocía el riesgo de transmisión materno-infantil de ambas entidades en más del $65,0 \%$ de los casos.

El $17,3 \%$ de las encuestadas estaba embarazada antes de salir de Venezuela, es decir, la mayoría $(82,6 \%)$ concibió durante el trayecto o después de haber llegado a Colombia. El $39,1 \%$ de las gestantes no había recibido control prenatal durante este embarazo, solo el 20,9\% inició controles en el primer trimestre de gestación, con un porcentaje mucho menor para las que llegaron al país antes de los tres meses de embarazo (Figura 2). Entre las que sí habían recibido, 1,8 fue el promedio controles prenatales ( $\mathrm{DE}=1,4$ controles).

Casi todas estaban consumiendo ácido fólico (96,3\%), mientras que hierro y calcio solo el $68,2 \%$ y el $57,8 \%$, respectivamente. De acuerdo con la ELCSA, la proporción de inseguridad alimentaria en el hogar fue del $87,5 \%$ (mayor en Barranquilla), mientras que, según la hemoglobina en sangre, el $51,3 \%$ de las valoradas tenía anemia (Tabla 3).

Durante los seis meses anteriores a la encuesta, el $25,9 \%$ de las mujeres requirieron atención sanitaria por urgencias independiente de la atención prenatal. La mayoría (7/10) cree que le brindaron la atención necesaria para su problema de salud, mientras que el $62,7 \%$ calificó la atención como muy buena o buena.

- CP en 1er trimestre $\quad \mathrm{CP}$ en 2do trimestre $\quad \mathrm{CP}$ en 3er trimestre $\quad$ Ningún control prenatal

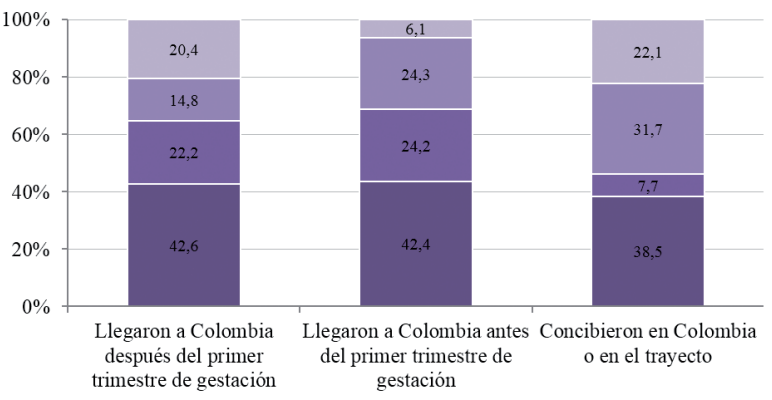

Figura 2. Trimestre de inicio del control prenatal según tiempo de migración a Colombia. Gestantes venezolanas migrantes en condición irregular en Barranquilla y Riohacha, 2018-2019.

\section{Violencia contra las gestantes}

Se les preguntó a las mujeres por la experiencia de diversos tipos de violencias perpetradas por su pareja o expareja durante la gestación actual (Tabla 3). Al respecto, se reportó principalmente violencia psicológica (89,0\%) (como celos, acuso de serle infiel, haberle impedido encontrarse con amigos, limitarle el contacto con familia, controlarle todo el tiempo o no consultarle sobre las decisiones importantes de la familia), seguido por la violencia económica $(31,4 \%)$ (como sentirse vigilada por él en la forma como gasta el dinero, haber sido amenazada con quitarle el apoyo económico, haberle prohibido trabajar, él se gastó el dinero que se necesitaba para el hogar, o haberle quitado dinero o bienes). Adicionalmente, un $7,1 \%$ dijo haber sido víctima de violencia física por parte de otra persona como padres, hermanos, tíos y amigos, entre otros. Las gestantes migrantes asentadas en Riohacha, reportaron mayores niveles de todos los tipos de violencia. 
Tabla 3. Características de salud en gestantes venezolanas migrantes en condición irregular en Barranquilla y La Guajira, 20182019.

\begin{tabular}{|c|c|c|c|c|c|c|c|c|c|}
\hline & \multicolumn{3}{|c|}{ Barranquilla } & \multicolumn{3}{|c|}{ Riohacha } & \multicolumn{3}{|c|}{ Total } \\
\hline & Comu & Hosp & Total & Comu & Hosp & Total & Comu & Hosp & Total \\
\hline \multicolumn{10}{|c|}{$\begin{array}{l}\text { Percepción de la salud durante el embarazo \% } \\
\end{array}$} \\
\hline Muy buena & 0,0 & 1,6 & 1,3 & 5,3 & 3,1 & 3,5 & 2,1 & 2,1 & 2,1 \\
\hline Buena & 57,6 & 62,3 & 61,4 & 28,9 & 36,8 & 35,3 & 46,4 & 52,4 & 51,3 \\
\hline Regular & 39,0 & 34,6 & 35,4 & 55,3 & 46,0 & 47,8 & 45,4 & 39,0 & 40,2 \\
\hline Mala & 3,4 & 1,6 & 1,9 & 10,5 & 12,3 & 11,9 & 6,2 & 5,7 & 5,8 \\
\hline Muy mala & 0,0 & 0,0 & 0,0 & 0,0 & 1,8 & 1,5 & 0,0 & 0,7 & 0,6 \\
\hline \multicolumn{10}{|c|}{ Nivel de inseguridad alimentaria en el hogar \% } \\
\hline Seguridad alimentaria & 11,9 & 8,9 & 9,5 & 7,9 & 19,3 & 17,2 & 10,3 & 13,0 & 12,5 \\
\hline Inseguridad leve & 37,3 & 41,2 & 40,5 & 21,1 & 20,5 & 20,6 & 30,9 & 33,1 & 32,7 \\
\hline Inseguridad moderada & 37,3 & 26,8 & 28,8 & 23,7 & 24,7 & 24,5 & 32,0 & 26,0 & 27,1 \\
\hline Inseguridad severa & 13,6 & 23,0 & 21,2 & 47,4 & 35,5 & 37,7 & 26,8 & 27,9 & 27,7 \\
\hline \multicolumn{10}{|l|}{ Anemia $\%$} \\
\hline Hemoglobina $<11 \mathrm{~g} / \mathrm{dl}$ & 51,6 & 51,2 & 51,3 & -- & -- & -- & -- & -- & -- \\
\hline \multicolumn{10}{|c|}{ Síntomas depresivos clínicamente significativos \% } \\
\hline Sí & 25,4 & 23,3 & 23,7 & 39,5 & 46,4 & 45,1 & 30,9 & 32,4 & 32,1 \\
\hline \multicolumn{10}{|l|}{ Conductas de riesgo $\%$} \\
\hline Cigarrillo & 1,7 & 0,0 & 0,3 & 5,3 & 3,6 & 3,9 & 3,1 & 1,4 & 1,7 \\
\hline Alcohol & 8,5 & 0,4 & 1,9 & 13,2 & 12,7 & 12,7 & 10,3 & 5,2 & 6,2 \\
\hline Drogas ilícitas & 1,7 & 0,4 & 0,6 & 0,0 & 0,6 & 0,5 & 1,0 & 0,5 & 0,6 \\
\hline \multicolumn{10}{|c|}{ Necesitó y recibió atención por urgencias además de las consultas prenatales \% } \\
\hline Sí & 35,6 & 37,6 & 37,3 & 10,5 & 7,8 & 8,3 & 25,8 & 25,9 & 25,9 \\
\hline \multicolumn{10}{|c|}{ Considera que le brindaron la atención necesaria $\%$ b } \\
\hline Sí \% & 66,1 & 75,5 & 73,7 & 75,0 & 66,7 & 69,8 & 66,7 & 74,5 & 73,1 \\
\hline \multicolumn{10}{|c|}{ Percepción de la calidad de la atención recibida $\%^{\mathrm{b}}$} \\
\hline Muy buena & 15,0 & 5,5 & 7,2 & 25,0 & 0,0 & 6,7 & 16,7 & 4,9 & 7,1 \\
\hline Buena & 40,0 & 58,2 & 55,0 & 25,0 & 72,7 & 60,0 & 37,5 & 59,8 & 55,6 \\
\hline Regular & 20,0 & 17,6 & 18,0 & 0,0 & 18,2 & 13,3 & 16,7 & 17,6 & 17,5 \\
\hline Mala & 20,0 & 18,7 & 18,9 & 50,0 & 9,1 & 20,0 & 25,0 & 17,6 & 19,0 \\
\hline Muy mala & 5,0 & 0,0 & 0,9 & 0,0 & 0,0 & 0,0 & 4,2 & 0,0 & 0,8 \\
\hline \multicolumn{10}{|c|}{ Violencia por parte de la pareja o expareja \% } \\
\hline Económica & 20,3 & 21,4 & 21,2 & 47,4 & 47,0 & 47,1 & 30,9 & 31,5 & 31,4 \\
\hline Sexual & 0,0 & 1,2 & 1,0 & 2,6 & 3,0 & 2,9 & 1,0 & 1,9 & 1,7 \\
\hline Física & 10,2 & 5,5 & 6,3 & 21,1 & 13,9 & 15,2 & 14,4 & 8,8 & 9,8 \\
\hline Psicológica & 88,1 & 87,1 & 87,3 & 86,8 & 92,8 & 91,7 & 87,6 & 89,3 & 89,0 \\
\hline \multicolumn{10}{|c|}{ Violencia por parte de otra persona $\%$} \\
\hline Física & 15,3 & 3,1 & 5,4 & 15,8 & 8,4 & 9,8 & 15,5 & 5,2 & 7,1 \\
\hline
\end{tabular}

a. Prueba de hemoglobina en sangre capilar a submuestra de 273 gestantes asentadas en Barranquilla b. Solo para aquellas que obtuvieron el servicio de urgencias cuando lo necesitó

\section{Discusión}

En síntesis, en este trabajo se encontró que las mujeres gestantes migrantes venezolanas se encuentran en su mayoría en un alto grado de vulnerabilidad social y económica, lo cual es coherente con su estatus migratorio irregular. Como resultados más relevantes, las gestantes migrantes venezolanas del estudio no tuvieron garantizado el acceso a la atención primaria, que incluyera los controles prenatales, la toma de complementos nutricionales y la protección social necesaria para disminuir el riesgo obstetrico y perinatal. Es importante, sin embargo, tener presente que las migrantes son un grupo heterogéneo, y que no todas 
necesariamente se encuentran en esta condición de vulnerabilidad, además que esta puede cambiar en la medida en que la regularización les permita acceder a trabajo, ingresos y seguridad social.

Con relación al acceso a servicios de salud, si bien el $96,3 \%$ dijo no estar afiliada al sistema de salud colombiano, el $25,9 \%$ de las encuestadas relató que cuando lo necesitó accedió efectivamente a los servicios de urgencias, de estas, la mayoría piensa que le brindaron la atención necesaria y valoraron positivamente la calidad del servicio. Esta atención ha sido posible gracias a la respuesta del Gobierno colombiano frente a los grupos prioritarios y vulnerables en el marco de la migración venezolana ${ }^{13-16}$ y a las acciones de organizaciones de cooperación internacional y ayuda humanitaria que han apoyado, entre otras, brigadas extramurales en diferentes territorios de acogida.

El plan de respuesta del sector salud al fenómeno migratorio ha garantizado en la teoría la atención integral de las gestantes en Colombia, independientemente de su estatus migratorio, así como también ha incorporado la necesidad de involucrarlas como blanco en los planes de intervención colectiva ${ }^{13}$. De tal modo que las migrantes venezolanas irregulares tienen derecho actualmente no solo a la atención de urgencias, parto y puerperio, sino también al control prenatal y a los beneficios de las acciones colectivas ${ }^{14,15}$. Sin embargo, a pesar de las resoluciones tomadas por las autoridades sanitarias a nivel nacional, estas medidas no se han traducido en todos los territorios del mismo modo en beneficios para la población migrante en medidas de promoción de la salud y la prevencion de la enfermedad, y así mismo, la atención estaría determinada por procedimientos administrativos no explícitos, así como por las limitaciones financieras para garantizar su atención en cada ente territorial. El gran esfuerzo del gobierno nacional y de las administraciones locales se limita a la atención en el servicio de urgencias, donde las migrantes tienen una buena percepción de los servicios de salud, sin embargo, sin cobertura en atención primaria, no existirá disminución de riesgo para la salud individual y colectiva para las gestantes migrantes, en temas como acceso oportuno y continuo a tratamientos y violencia de género, ni se verán los beneficios sociales de la promoción de la agencia y empoderamiento, necesarias para que tanto las madres como las generaciones que nacen en esta ola migratoria tengan mayores oportunidades de bienestar.

En este sentido, debe prestarse atención al acceso, oportunidad, continuidad e integridad del control prenatal; entre los hallazgos de este estudio está la falta de oportunidad diagnóstica para eventos prioritarios en salud pública como el VIH/SIDA y la Sifilis, entre otros, que sin intervención oportuna, se convierten en eventos catastróficos para el individuo y para la sociedad.

Es importante resaltar que el estado de salud de las mujeres migrantes embarazadas no solo se relaciona con sus condiciones de vida en Colombia al momento de la encuesta, sino que también influyen sus características individuales, las condiciones de vida anteriores, la red social en Venezuela y las condiciones del viaje, es decir, influyen de manera importante los determinantes sociales tanto del origen, como del tránsito y destino.

Entre las problemáticas prioritarias identificadas en esta investigación, se encuentran las carencias nutricionales, cuatro de cinco hogares tenía inseguridad alimentaria y la mitad de la submuestra presentó anemia. La inseguridad alimentaria ha sido consistentemente asociada con peores resultados en salud en los diferentes momentos del ciclo vita ${ }^{17}$. Si bien el instrumento utilizado fue diferente, los resultados de este estudio se asemejan a los de Omidvar, et.al. ${ }^{18}$ quienes encontraron inseguridad alimentaria en el $77,0 \%$ de los inmigrantes afganos en dos ciudades de Irán en 2010. Por su parte, según Noran, et. al. ${ }^{19}$ la deficiencia de hierro durante la gestación es un problema de salud global, que afecta cerca del $52,0 \%$ de las mujeres embarazadas en el mundo en desarrollo, una proporción similar a la encontrada entre las gestantes de este estudio $(51,3 \%)$.

Por su parte, de acuerdo con Miszkurka, et. al. las mujeres migrantes presentan mayor prevalencia de síntomas depresivos clínicamente significativos durante la gestación y el periodo posparto ${ }^{20}$. En un estudio adelantado en embarazadas de 18 a 45 años en Barranquilla (2012), Borda, et.al. reportaron depresión en el $19,2 \%$ de las gestantes ${ }^{21}$, una cifra menor a la encontrada en este estudio (32,1\%). Por su parte, en una investigación realizada en migrantes embarazadas reclutadas en dos hospitales de Montreal (Canadá) ${ }^{22}$, hallaron un $42,0 \%$ con síntomas depresivos, $\mathrm{y}$ asociación estadística con deficiencias funcionales, síntomas somáticos, ausencia de apoyo social, eventos vitales estresantes y peor calidad marital (consenso, satisfacción, cohesión y expresiones de afecto). Valga advertir que en ambos estudios utilizaron escalas diferentes para valorar la depresión.

Con respecto a la violencia perpetrada por la pareja, esta investigación reporta un porcentaje superior al rango encontrado en otras investigaciones en Europa y América (17,0\% y 70,5\%) de acuerdo con la revisión de literatura publicada por Gonçalves y 
Matos en relación con este tema ${ }^{23}$. Este ha sido el tipo de violencia más estudiada entre mujeres migrantes y es pertinente señalar que se requieren estudios sobre otros tipos de agresiones menos visibles a las que están sujetas las mujeres migrantes en sus familias, comunidades, lugares de trabajo, espacios públicos e instituciones.

La Guajira y el Atlántico son dos de los cuatro departamentos con mayor proporción de migrantes irregulares del país, lo que hace del Caribe colombiano la región de mayor acogida de migrantes venezolanos irregulares junto con el noroccidente colombiano. Sin embargo, a pesar de las directrices nacionales, las respuestas en Salud Pública han sido diversas entre los entes territoriales que explicarían diferencias en el estado de salud de las gestantes migrantes, sumado a las diferencias en el perfil migratorio (pendular en Riohacha y con intención de permanencia en Barranquilla).

Entre las limitaciones del estudio se encuentra la imposibilidad de generalización a todas las mujeres migrantes gestantes procedentes de Venezuela. De acuerdo con el diseño muestral, los resultados pueden ser atribuidos a las mujeres encuestadas, todas ellas en condición irregular; además, debido a los tipos de muestreo, no pueden estimarse directamente algunos parámetros como el acceso efectivo a los servicios de salud (entre tanto la captación de la mayoría se hizo en un ambiente hospitalario, es decir, ya habían accedido al sistema), además de las limitaciones propias del tipo de muestreo no probabilístico en bola de nieve ${ }^{24}$, entre los que se destaca la mayor probabilidad de encontrar resultados similares entre los participantes referidos. El diseño planteado en este estudio fue la mejor alternativa disponible dada la inexistencia de un marco muestral que cubra todo el universo y la alta movilidad de la población irregular, todos los días llegan nuevas personas y otras salen del país, y muchos de estos movimientos no se registran en los sistemas de información. Adicionalmente, se considera que se capta las principales fuentes de heterogeneidad de la población a través de la búsqueda y el análisis en forma estratificada en el contexto comunitario y hospitalario, como también por ciudad. Finalmente, es razonable suponer que la población blanco de interés: gestantes migrantes irregulares, sí está adecuadamente representada por la muestra, esto en razón a que se trata de una población heterogénea en sus condiciones sociales y económicas y en otros determinantes sociales que explicarían que las condiciones de salud tengan un comportamiento similar a lo observado en el Registro
Administrativo de Migrantes Venezolanos (RAMV), o en las bases de datos de registro de atenciones de ambas ciudades, en donde varios parámetros como edad y escolaridad son prácticamente iguales que los estimadores obtenidos en la muestra de este trabajo. Estimaciones más precisas se encontrarán en los análisis posteriores cuando se termine el reclutamiento en un año, pero dada la coyuntura actual estos resultados debían ser publicados con oportunidad, y es probable que los estimadores puntuales de resultados finales varíen poco.

Este reporte tiene diversas fortalezas, entre ellas y de acuerdo con nuestro conocimiento, se constituye en el primer informe con fuentes primarias en el que se develan las condiciones demográficas, sociales, económicas, de estado de salud y experiencia en el acceso a los servicios sanitarios de las mujeres gestantes migrantes irregulares asentadas en las principales ciudades receptoras del Caribe colombiano. Valga mencionar que las migrantes irregulares se han identificado como una población particularmente vulnerable, algunas veces invisibilizadas y expuestas a diferentes tipos de abuso ${ }^{25,26}$ que requiere especial atención y que la región Caribe es uno de los territorios con mayor recepción de migrantes irregulares venezolanos ${ }^{27}$. En este sentido, es probable que la regularización les permita acceder a mejores fuentes de ingreso, y, por lo mismo, ser un factor clave para la mejora progresiva del estado de salud de las gestantes migrantes y de los migrantes en general.

Si bien los hallazgos del estudio develan diversas condiciones de vulnerabilidad socio-económica entre las gestantes migrantes venezolanas, es importante resaltar que estos no se alejan de las condiciones de la población colombiana pobre no asegurada, siendo probable que enfrenten en principio las mismas dificultades de acceso al sistema de salud. Por otro lado, la capacidad institucional de cada ente territorial, con el apoyo del gobierno nacional, será determinante para garantizar el goce efectivo del derecho a la salud de las gestantes migrantes irregulares. Se espera que los resultados de esta investigación aporten a la ejecución de medidas específicas de protección y atención a las migrantes gestantes, y ayude indirectamente a la superación de la vulnerabilidad. Aunque de forma más general, es probable que más allá del sistema de salud, sea principalmente la regularización, en cuanto puerta de acceso al trabajo formal, ingreso y aseguramiento social, la que permita modificar realmente los determinantes sociales de la salud de estas mujeres. 


\section{Agradecimientos}

A Maolys Jiménez, quien coordinó el trabajo de campo; a la estudiante de maestría Kellys Quintana, por su apoyo logístico en la Guajira. A la Fundación "De pana que sí" liderada por Thailer Fiorillo y Maryanella Sánchez, y a todas las mujeres migrantes y gestantes que aceptaron participar en nuestro estudio. A la Dirección de Investigación, Desarrollo e Innovación de la Universidad del Norte por la financiación de este proyecto.

\section{Consideraciones éticas}

Los principios éticos estuvieron siempre en concordancia con la declaración de Helsinki y la Resolución 8430 de 1993 de Colombia. Esta investigación fue clasificada como con riesgo menor al mínimo y avalada por el Comité de Ética de la División de Salud de la Universidad del Norte. Todas las gestantes firmaron el consentimiento informado y siempre se respetó su autonomía para participar en el estudio, indicando que su participación sería voluntaria y sin perjuicio si decidían no participar. A todas las participantes se les brindó orientación para acceder al sistema de salud, sensibilización sobre signos de alarma y recomendaciones generales para el cuidado durante el embarazo.

\section{Conflicto de interés}

Los investigadores no reportan conflictos de interés.

\section{Referencias}

1. Ministerio de Salud. Seguimiento estadístico $\mathrm{N}^{\circ} 26$. Migrantes Venezolanos en Colombia. Corte 31 de diciembre de 2018; 2019.

2. Organización Internacional para las Migraciones. Informe sobre las migraciones en el mundo. Los migrantes y las ciudades: Nuevas colaboraciones para gestionar la movilidad Organización Internacional para las Migraciones (OIM); 2015. 247 p.

3. Instituto Nacional de Salud. Boletín $\mathrm{N}^{\circ} 16$. Notificación eventos de interés en salud pública durante. Fenómeno migratorio; 2019.

4. Ministerio de Saludy Protección Social. Decreto 1768 de 2015. Por el cual se establecen las condiciones para la afiliación al Sistema General de Seguridad Social en Salud de los migrantes colombianos que han sido repatriados, han retornado voluntariamente al país o han sido deportados o expulsado de la República Bolivariana de Venezuela; 2015. 4p
5. Ministerio de Salud y Protección Social. Presidencia de la República de Colombia. Decreto 866 de 2017. Por el cual se sustituyen apartados del decreto 780 de 2016 en cuanto al giro de recursos para la atención inicial de urgencias a migrantes; 2017 p. 2016-8.

6. Rodríguez Valladolid N. Género, inmigración e intervención social. Oriente y occidente: la construcción de la subjetividad femenina. 2014; p. $79-100$.

7. Organizacion Internacional para las Migraciones. Las Migraciones en America Latina y el Caribe; 2005.

8. Palacios Valencia Y. Perspectiva de género en los fenómenos migratorios: estudio desde Europa y América Latina. CES Derecho. 2016; 7(2): 145-162.

9. Álvarez M, Vélez O. Adaptación y validación interna y externa de la Escala Latinoamericana y el Caribe para la Medición de Seguridad Alimentaria en el Hogar ELCSA- Colombia. Componente Adaptación lingüística de la ELCSA. Universidad de Antioquia; 2008.

10. Organización de las Naciones Unidas para la Alimentación y la Agricultura (FAO), Comité científico de la ELCSA. Escala Latinoamericana y Caribeña de Seguridad Alimentaria (ELCSA): Manual de uso y aplicaciones. Roma: FAO; 2012. $78 \mathrm{p}$.

11. Gempp Fuentealba R, Thieme C. Efecto de diferentes métodos de puntuación sobre la fiabilidad, validez y puntos de corte de la escala de depresión del Centro para Estudios Epidemiológicos (CES-D). Terapia Psicologica. 2010; 28(1): 5-12. doi: http:// dx.doi.org/10.4067/S0718-48082010000100001.

12. OMS. Concentraciones de hemoglobina para diagnosticar la anemia y evaluar su gravedad (WHO/ NMH/NHD/MNM/11.1). Ginebra. OMS; 2011. 7 p.

13. Ministerio de Salud y Protección Social. Plan de Respuesta del Sector Salud al fenómeno migratorio. República de Colombia; 2017. 75 p.

14. Ministerio de Salud y Protección Social. Circular 25 de 2017. Asunto: Fortalecimiento de acciones en salud pública para responder a la situación de migración de población proveniente de Venezuela. República de Colombia; 2017. 7 p.

15. Presidencia de la República. Decreto 1770 de 2015. Por el cual se declara el Estado de Emergencia Económica, Social y Ecológica en parte del territorio nacional. República de Colombia; 2015. 13 p.

16. Consejo Nacional de Política Económica y Social, DNP. Documento Conpes 3950. Estrategia para la atención de la migración desde Venezuela. DNP; 2018. $115 \mathrm{p}$.

17. FAO, FIDA, Programa Mundial de Alimentos. El estado de la inseguridad alimentaria en el mundo, 
2015: cumplimiento de los objetivos internacionales para 2015 en relación con el hambre: balance de los desiguales progresos. 1a ed. Vol. 1. Roma: FAO; 2015. $66 \mathrm{p}$.

18. Omidvar N, Ghazi-Tabatabie M, Sadeghi R, Mohammadi F, Abbasi-Shavazi MJ. Food Insecurity and its sociodemographic correlates among Afghan immigrants in Iran. J Health Popul Nutr. 2013; 31(3): 356-366.

19. Noran A, Mohammed J. The impact of maternal iron deficiency and iron deficiency anemia on child's health. Saudi Med J. 2015; 36(2): 146-149. doi: http://doi.org/10.15537/smj.2015.2.10289.

20. Miszkurka M, Goulet L, Zunzunegui MV. Contributions of immigration to depressive symptoms among pregnant women in Canada. Can J Public Health. 2010; 101(5): 358-364. doi: https://doi.org/10.1007/BF03404853.

21. Borda Pérez MA, Forero C, Ahcar Cabarcas N, Hinestrosa C, Polo Lobo S, Staaden Garavito M, et al. Depresión y factores de riesgos asociados en embarazadas de 18 a 45 años asistentes al Hospital Niño de Jesús en Barranquilla (Colombia). Rev Cient Salud Uninorte. 2013; 29(3): 394-405.

22. Zelkowitz P, Schinazi J, Katofsky L, Saucier JF, Valenzuela M, Westreich R, et al. Factors associated with depression in pregnant immigrant women. Transcult Psychiatry. 2004; 41(4): 445-464. doi: http://doi.org/10.1177/1363461504047929.

23. Gonçalves M, Matos M. Prevalence of violence against immigrant Women: A systematic review of the literature. J Fam Viol. 2016; 31(6): 697-710. doi: http://doi.org/10.1007/s10896-016-9820-4.

24. Bagheri A, Saadati M. Exploring the effectiveness of chain referral methods in sampling hidden populations. Indi J Science Technol. 2015; 8(30): 1-8. doi: http://doi.org/10.17485/ijst/2015/v8i30/84754.

25. Bloch A, Chimienti M. Irregular migration in a globalizing world. Irregular Migrants. 2013; 34(8): 1271-1285. doi: https://doi.org/10.1080/01419870.2011.560277.

26. Ambrosini M. Irregular migration and invisible welfare. 1a ed. London: Palgrave Macmillan UK; 2013.

27. Consejo Nacional de Política Económica y Social. República de Colombia. Departamento Nacional de planeación. Documento Conpes 3950. Estrategia para la atención de la migración desde Venezuela; 2018. p. 1-115. 